\title{
Protocolos na enfermagem: relato de experiência de uma disciplina sobre tecnologias em saúde
}

\author{
Nursing guidelines: experience report of a subject on health technologies
}

Protocolos en enfermería: relato de experiencia de una disciplina en tecnologías sanitarias

Anna Gabriela Cavalcanti Arais ${ }^{1 *}$, Vitória Silva da Rosa1 ${ }^{1}$, Victória Tiyoko Moraes Sakamoto ${ }^{1}$ Carine Raquel Blatt", Rita Catalina Aquino Caregnato'.

\section{RESUMO}

Objetivo: Relatar a experiência de enfermeiras sobre o conhecimento construído em uma disciplina de tecnologias em saúde, com enfoque na tecnologia leve-dura dos protocolos existentes. Relato de experiência: Uma Universidade Federal brasileira oferece uma disciplina sobre tecnologias da saúde, abordando tipos existentes, sua avaliação e aplicação no cenário da saúde. Essa temática envolve a construção do saber fundamentado na prática baseada em evidências, utilizando a tecnologia como coadjuvante no processo educacional e assistencial dos profissionais da saúde. Para enfermeiros, a disciplina viabiliza novas perspectivas acerca das práxis diárias, pois promove conhecimentos específicos e propicia uma visão crítica sobre processos de trabalho. Além disso, a aplicação dos conhecimentos adquiridos na disciplina fomenta o desenvolvimento de protocolos e ferramentas que auxiliam nas tomadas de decisão, qualificando a assistência. Protocolos são tecnologias leve-duras fundamentadas em evidências científicas que oportunizam a assistência, a educação e o empoderamento dos profissionais da saúde. Considerações finais: Há modelos distintos de protocolos, categorizados conforme o conteúdo e enfoque prático, sendo sua aplicabilidade o maior diferencial entre eles. O Protocolo Assistencial vem sendo o instrumento mais utilizado para promover a enfermagem baseada em evidências, permitindo ao enfermeiro implementar o conhecimento científico na assistência, qualificando-a e tornando-a segura.

Palavras-chave: Enfermagem, Protocolos, Tecnologias em saúde.

\begin{abstract}
Objective: To report the experience of nurses on the knowledge built in a discipline of health technologies, focusing on the light-hard technology of existing guidelines. Experience report: A Brazilian Federal University offers a discipline that discusses health technologies, addressing the existing types, their evaluation and application in the health scenario. This theme involves the construction of knowledge based on evidencebased practice, using technology as a support in the educational and care process of health professionals. For nurses, the discipline enables new perspectives on daily praxis, as it promotes specific knowledge and provides a critical view of work processes. In addition, the application of knowledge acquired in the discipline encourages the development of guidelines and tools that help in decision-making, qualifying assistance. Guidelines are classified as light-hard technologies based on scientific evidence that provide assistance, education and the empowerment of health professionals. Final considerations: There are different models of guidelines, which are categorized according to content and practical focus, with their applicability being the biggest difference between them. The Care Guidelines has been the most used instrument to promote evidence-based nursing, allowing nurses to implement scientific knowledge in professional care, qualifying it and making it safe.
\end{abstract}

Key words: Nursing, Guidelines, Health technologies.

${ }^{1}$ Universidade Federal do Rio Grande do Sul (UFCSPA), Porto Alegre - RS.

*E-mail: anna.arais@ufcspa.edu.br

SUBMETIDO EM: 7/2021

ACEITO EM: 7/2021

PUBLICADO EM: 8/2021 


\section{RESUMEN}

Objetivo: Informar la experiencia de los enfermeros sobre el conocimiento construido en una disciplina de tecnologías de la salud, enfocándose en la tecnología liviana de los protocolos existentes. Informe de experiencia: Una Universidad Federal de Brasil ofrece una disciplina que discute las tecnologías de la salud, abordando los tipos existentes, su evaluación y aplicación. Este tema involucra la construcción de conocimiento a partir de la práctica basada en la evidencia, utilizando la tecnología como soporte en el proceso educativo y asistencial de los profesionales. La disciplina permite perspectivas sobre la praxis diaria, promoviendo conocimientos y proporcionando una visión de los procesos de trabajo. Además, los conocimientos adquiridos fomentan el desarrollo de protocolos y herramientas que ayuden en la toma de decisiones, habilitando la asistencia. La clasificación de protocolos en tecnologías blandas y duras basadas en evidencia científica brinda asistencia, educación y capacitación a los profesionales de la salud. Conclusión: Existen diferentes protocolos, que se categorizan según su contenido y enfoque práctico, siendo su aplicabilidad la diferencia entre ellos. El Protocolo de Atención ha sido el instrumento utilizado para promover la enfermería basada en la evidencia, permitiendo al enfermero implementar el conocimiento científico en el cuidado, calificándolo y haciéndolo seguro.

Palabras clave: Enfermería, Protocolos, Tecnologías sanitarias.

\section{INTRODUÇÃO}

Tecnologia em Saúde (TS) pode ser definida como um conjunto de instrumentos materiais e não-materiais que auxiliam na prática assistencial (GONÇALVES GAA, et al., 2020). Existem diferentes abordagens em relação a sua classificação. De acordo com Merhy EE (2017), as TS classificam-se em: duras, leve-duras e leves. A tecnologia dura refere-se aos recursos materiais como equipamentos e máquinas; leve-dura são os saberes estruturados podendo ser tecnológicos clínicos e epidemiológicos, como os instrumentos educacionais; e leve são as relações de agir na produção dos atos de saúde, envolvendo o acolhimento e humanização do cuidado (SILVA MCDA, et al., 2017). Outra classificação refere-se às tecnologias de processo e as de produto, uma vez que engloba desde teorias e processos educativos até a criação de um bem palpável como medicamentos e equipamentos (SANTOS NO, et al., 2016).

A Prática Baseada em Evidência (PBE), é uma abordagem com novo paradigma na área da saúde, aproximando a pesquisa científica da prática assistencial. Consiste na escolha da melhor evidência científica disponível, associada à expertise do profissional e as preferências do paciente para a tomada de decisão assistencial, qualificando o cuidado prestado (FERRAZ L, et al., 2020).

A PBE iniciou no Canadá, em meados de 1980, na área médica, com objetivo da promoção da melhor assistência à saúde e a especialização do ensino, posteriormente tornando-se uma prática multidisciplinar (VIEIRA TW, et al., 2020). A incorporação da PBE gera maior qualidade aos cuidados prestados, diminuindo a variabilidade das ações e custos, melhorando os resultados clínicos, contribuindo para os serviços de saúde alcançarem a eficácia e a segurança nas práticas (SAUNDERS H e VEHVILÄINEN-JULKUNEN K, 2017; CARDOSO DFB, et al., 2020).

A enfermagem trabalha continuamente para incorporar as evidências científicas na sua prática assistencial, evoluindo do trabalho empírico para um cuidado fundamentado em evidências, buscando promover práticas seguras e efetivas para o cuidado do paciente (VIEIRA TW, et al., 2020; CATUNDA HLO, et al., 2017). Nos últimos anos, cresceu a atuação do enfermeiro na elaboração, aplicação e avaliação das TS, com destaque para a produção de protocolos e materiais educacionais fundamentados na PBE (MORAES LMS, et al., 2016). A produção fundamentada na PBE para a enfermagem, na língua inglesa, encontra-se nos guidelines, entretanto no Brasil essa produção apresenta-se com diferentes denominações, como Diretrizes Clínicas e Protocolos, podendo os últimos ser Assistenciais ou Clínicos ou de Uso. Além disso, existem ainda na prática clínica o Procedimento Operacional Padrão (POP) (CATUNDA HLO, et al., 2017; PIMENTA C, et al., 2015). 
Ao investigar na literatura nacional a produção científica sobre tecnologias leve-duras, identificaram-se diferentes tipos de protocolos, com diferentes aplicações e conceitos. Ao cursar uma disciplina de TS, as autoras decidiram compartilhar os conhecimentos adquiridos para esclarecer diferenças existentes e contribuir para a prática da enfermagem. Dessa forma, traçou-se como questão norteadora para este artigo: quais são os tipos e particularidades dos protocolos utilizados pela equipe de enfermagem? Alinhado a essa questão, definiu-se como objetivo deste artigo: relatar a experiência de enfermeiras sobre o conhecimento construído em uma disciplina de TS com enfoque na tecnologia leve-dura dos protocolos existentes.

\section{RELATO DE EXPERIÊNCIA}

Uma Universidade Federal, localizada na região sul do Brasil, oferta cursos exclusivos na área da saúde, oferecendo duas vezes por ano uma disciplina denominada "Tecnologias em saúde: evidências científicas para a aplicação na prática". Disponibilizada para os Programas Stricto Sensu, tem a finalidade de apresentar aos profissionais da saúde como o Brasil está estruturado para a avaliação, incorporação e uso de tecnologias nos diferentes cenários de saúde.

Seu objetivo é promover a reflexão sobre uso da TS e aplicação das evidências científicas na prática. A abordagem introduz a temática das TS como coadjuvante no processo educacional e assistencial dos profissionais da saúde. Tem carga horária de 30 h/a e seu conteúdo programático está distribuído em sete encontros, seguindo uma sequência lógica na abordagem dos temas.

O primeiro encontro introduz a temática sobre as TS, trazendo os conceitos de tecnologias, apresenta a Política Nacional de Gestão de Tecnologias em Saúde e sua inserção no Sistema Único de Saúde (SUS), a Rede Brasileira de Avaliação de Tecnologias em Saúde (REBRATS) e os Núcleos de Avaliação de Tecnologias em Saúde (NATS) existentes no país.

O encontro seguinte abrange a PBE, introduzindo a temática das diretrizes e protocolos, como desenvolvêlos, avaliar a qualidade das evidências selecionadas e a força de recomendação a partir do sistema Grading of Recommendations Assessment, Development and Evaluation (GRADE), bem como a avaliação da qualidade metodológica de uma diretriz ou de um protocolo a partir do instrumento Appraisal of Guidelines for Research \& Evaluation (AGREE II). Aula de grande relevância para os profissionais da saúde, uma vez que introduz a temática das PBE na condução da busca pelas melhores evidências disponíveis para as tomadas de decisão na prática cotidiana. Além disso, apresenta ferramentas capazes de fortalecer, sustentar e qualificar as ações do profissional, independente da área de conhecimento e/ou atuação. Dentre essas ferramentas, destacam-se os tipos de protocolos existentes, a saber: protocolo clínico, protocolo assistencial e protocolo de uso.

Nos três encontros seguintes ocorre a imersão do aluno no aprofundamento do conteúdo da disciplina, realizando atividades que consolidem a construção do conhecimento. Realizadas leituras, síntese dos capítulos de um manual de avaliação de TS, avaliação de uma diretriz ou protocolo a partir do instrumento AGREE II para apresentação e discussão coletiva.

Nos dois últimos encontros apresenta a temática da avaliação econômica das TS, com discussão sobre a importância e relevância dos desfechos em saúde, tipos de custos e como identificá-los, bem como a apresentação das avaliações de custo-efetividade e custo-utilidade. Os alunos realizam a elaboração de um projeto de avaliação econômica de uma tecnologia aplicada a sua realidade.

Ao término da disciplina, os discentes entraram em contato com os princípios gerais de avaliação de TS e os métodos de avaliação econômica. Compreendem como são realizadas as avaliações de TS e conhecem um pouco sobre diferentes cenários: sistemas organizacionais, educacionais, de informação e suporte, a partir de programas, protocolos (assistenciais, clínico e de uso) e procedimentos técnicos relacionados não apenas a equipamentos, mas também medicamentos e ferramentas de saúde. Espera-se com esta proposta metodológica estimular os discentes a refletir sobre a importância e o impacto dessas tecnologias na área da saúde. 


\section{DISCUSSÃO}

As TS fundamentadas na PBE impactam positivamente o processo de trabalho dos profissionais da saúde através da cientificidade da assistência, com as premissas das melhores práticas e segurança do paciente, qualificando o atendimento ao indivíduo, família e comunidade (JUNIOR CL, 2020). A enfermagem utiliza inúmeras TS durante a sua prática assistencial, sendo os protocolos os recursos mais utilizados (CATUNDA HLO, et al., 2017)

O Ministério da Saúde (MS) criou em 2011, através da Portaria oㅜ 2.915, a REBRATS como uma estratégia de disseminação da pesquisa, política e gestão das TS no Brasil. A REBRATS possui o propósito de subsidiar decisões de incorporação, monitoramento e abandono de tecnologias no contexto de suas utilizações nos sistemas de saúde, contribuindo para a formação e educação continuada na área. Constituída pelos NATS, reúne recursos e profissionais com competência técnica para desenvolver, promover e executar a avaliação das TS (BRASIL, 2011).

A Comissão Nacional de Incorporação de Tecnologias no Sistema Único de Saúde (CONITEC) tem a definição de dois termos para os documentos elaborados, Protocolos Clínicos e Diretrizes Terapêuticas (PCDT) e as Diretrizes Diagnósticas e Terapêuticas (DDT). São construídos visando a garantia das melhores práticas em saúde para diagnóstico, tratamento e acompanhamento dos pacientes assistidos pelo SUS. Os PCDT são documentos que estabelecem desde os critérios para o diagnóstico da doença ao melhor tratamento indicado baseado nos princípios de eficácia, segurança, efetividade e custo-efetividade. Já os DDT são voltados à área oncológica, norteando as melhores condutas baseadas nas pesquisas científicas. Devido ao financiamento diferenciado para as práticas oncológicas, esses documentos não se restringem às tecnologias incorporadas no SUS (BRASIL, 2021).

Entende-se como Diretrizes, o conjunto de recomendações técnicas e organizacionais construídas por especialistas, de grande renome na área de atuação, direcionadas para problemas específicos, de abrangência nacional que fundamentam legalmente os demais documentos na prática assistencial em saúde (BRASIL, 2016). A partir dessas definições técnicas, constituídas pelo MS, os demais documentos são construídos e inseridos nos serviços de saúde pelas mais diversas categorias profissionais. Através das diretrizes e incorporação das TS, os Conselhos Federativos dos profissionais de saúde podem recomendar e emitir normas que orientam a elaboração de diretrizes e protocolos para operacionalizar o cuidado em saúde realizado pelos profissionais, assim como conceituar e classificar os diferentes documentos (COFEN, 2018).

Baseados nos preceitos das diretrizes nacionais, na área da enfermagem, os protocolos são recomendações sistemáticas, fundamentados em evidências científicas que orientam decisões de profissionais a respeito dos cuidados a serem prestados na assistência à saúde, necessitando seguir um percurso metodológico rigoroso para sua construção (COFEN, 2018). Os enfermeiros podem utilizar os protocolos assistencial, clínico e de uso. Apesar das singularidades entre eles, os aspectos comuns a todos são: instrumentos educativos, busca de definição de padrões e ampliação da cultura de qualidade nos cuidados da enfermagem (LEMOS CS, et al., 2017).

O protocolo assistencial qualifica a assistência a partir da PBE ao instrumentalizar a tomada de decisão dos enfermeiros após julgamento clínico, com enfoque na assistência que deve ser prestada para atender as necessidades de saúde do paciente (PIMENTA C, et al., 2015). Indicado para apoiar o profissional no cuidado prestado como higiene e conforto, cuidados com lesões de pele, assim como garantir as funções privativas do enfermeiro na prática em saúde (COFEN, 2018; BITENCOURT JVOV, et al., 2020).

Esses protocolos possuem bases cientificamente comprovadas que garantem respaldo legal e otimização da assistência da enfermagem (SCHWEITZER G., et. al 2020; PAIXÃO DPSS et.al 2018). Os resultados da utilização dos protocolos assistenciais pelo enfermeiro têm demonstrado que se trata de uma ferramenta moderna, permitindo que todos os profissionais prestem cuidado padronizado para a segurança do paciente, de acordo com princípios técnico-científicos, contribuindo para diminuir as distorções adquiridas na prática, com finalidade educativa (FERNANDES MA, et al 2019). Apesar de ser construído por distintas metodologias e por diferentes instituições de saúde, o conteúdo abordado não permite variação de técnicas científicas entre 
as instituições de saúde, pois seu fundamento são as PBE previamente definidas na literatura, sendo readaptado, após validação de seu conteúdo, por especialistas, enriquecido através de visões, culturas e conhecimento científico (SANTOS NO, et al., 2020; CATUNDA HLO, et al., 2017).

Com enfoque maior nas políticas públicas de saúde, encontra-se o protocolo clínico, de escopo médico, definindo fluxos e algoritmos, com orientações de exames e medicamentos de escolha para determinado diagnóstico ou condição de saúde e doença (CARVALHO LR e ZEM-MASCARENHAS SH, 2020). Usado para padronizar a assistência aos pacientes com diagnósticos sugeridos ou definidos, tem o objetivo reduzir tempo de início de manejo específico ao paciente, para melhor prognóstico e redução de custos nas instituições de saúde (DOMINGUES CSB, et al., 2020). Um exemplo é o Protocolo Clínico da Sepse, que visa a rápida identificação dessa condição grave de saúde, aplicando a melhor evidência científica durante o atendimento no território nacional, iniciando o tratamento o mais breve possível, diminuindo a mortalidade e a morbidade associada à doença (RIBEIRO LL, 2020). Nele são definidos sinais e sintomas sugestivos das condições de saúde, exames a serem realizados e início imediato de antibióticos, reduzindo o tempo entre o diagnóstico e o tratamento indicado, devido às intervenções precoces, além da uniformidade das ações assistenciais das equipes médicas e de enfermagem (COREN-SP, 2018).

O protocolo de uso é definido como documento normativo que estabelece critérios e padrões para a utilização de uma tecnologia específica em determinada condição ou patologia (BRASIL, 2020). O crescente aumento da incidência de doenças crônico-degenerativas e o avanço nos tratamentos médicos e a incorporação de aparatos tecnológicos tem alterado o perfil dos doentes no Brasil (BRASIL, 2016). O governo brasileiro vem buscando alternativas para reorganizar e construir um novo modelo assistencial, que garanta segurança aos dependentes da utilização de tecnologias necessárias para a manutenção da vida (LANDEIRO MJL, et al., 2016)

Os pacientes dependentes de tecnologia são definidos como os que necessitam do uso por curto período, durante a reabilitação ou de forma permanente de dispositivos tecnológicos que auxiliam nas funções vitais, ou seja, não possuem condições clínicas de sobreviver sem o aparato tecnológico (SANTOS VT e MINAYO MCS, 2020). Com o objetivo de auxiliar o uso dessas tecnologias são construídos os Protocolos de Uso. Os modelos existentes e validados definem a patologia ou condição de saúde, os procedimentos invasivos indicados, conceito do aparato tecnológico abordado, cuidados que devem ser ofertados ao paciente e o acompanhamento por equipe multidisciplinar necessário (BRASIL, 2020).

O POP objetiva alcançar uniformidade na execução de uma tarefa prática, descrevendo passo a passo as atividades executadas em um setor da organização, sendo esse o modelo mais antigo utilizado pela enfermagem (TEIXEIRA SIN, et al., 2020; MACHADO LDS, et al., 2020; ALMEIDA LP, et al., 2017). Deve ser atualizado sempre que necessário, de acordo com princípios científicos a ser seguidos por todos os profissionais de forma padronizada (GOMES PPS, et al., 2018; PEREIRA LR, et al., 2017). Por se tratar de um instrumento interno, não há necessidade de um rigor científico ou percurso metodológico aprofundado, podendo ser construído pela própria equipe de enfermagem que irá praticá-lo (SALES CB, et al., 2018; BITENCOURT JVOV, et al., 2020; CRUZ DRS, et al., 2019). O POP permite que cada organização de saúde possa desenvolver suas tarefas de forma personalizada, usando recursos humanos e insumos que são disponíveis a sua realidade.

As TS, desde a abordagem teórica à materialização de um produto palpável, visam a prevenção, tratamento ou reabilitação das condições de saúde do paciente. Nesse contexto, os protocolos são as tecnologias leve-duras que permitem ao profissional de saúde assistir ao paciente com segurança, aplicando as melhores práticas em saúde além do cunho educacional presente nesse processo. Ao analisar os tipos de protocolos existentes, percebeu-se que os modelos de Protocolos Clínicos e de Uso, são de caráter e aplicação mais direcionados à área médica, pois definem e descrevem o uso de medicamentos, além de indicações de exames e intervenções cirúrgicas. Da mesma forma, na enfermagem o Protocolo Assistencial tem sido o instrumento mais usado na construção do conhecimento e suporte teórico à assistência profissional, uma vez que instrumentaliza os cuidados de enfermagem específicos às necessidades do paciente. 
A disciplina com abordagem nas TS contribuiu para o desenvolvimento do conhecimento acerca das tecnologias leve-duras, permitindo diferenciar os tipos de protocolos existentes e o seu impacto na prática assistencial. Na busca da qualificação e segurança na assistência em saúde, conhecer e utilizar protocolos são fundamentais para o cuidado baseado em evidência. Destaca-se a importância ao elaborar ou ler um protocolo, de conhecer os diferentes tipos existentes para que seja possível reconhecer as principais particularidades e indicações de cada um, facilitando ao profissional a escolha do tipo mais indicado de acordo com seu objetivo. A construção, validação e implementação de protocolos na enfermagem tem colaborado para instrumentalizar o enfermeiro nas atividades de assistência, gestão e educação, promovendo um modelo de implementação do conhecimento científico na assistência profissional.

\section{REFERÊNCIAS}

1. ALMEIDA LP, et al. A não utilização dos procedimentos operacionais padrão por profissionais de saúde em um centro de diálise. Revista Enfermagem Atual In Derme, 2017.

2. BITENCOURT JVOV, et al. Protagonismo do Enfermeiro na estruturação e gestão de uma Unidade específica para Covid-19. Texto\& contexto - enferm., 2020; 29: e20200213.

3. BRASIL. Ministério da Saúde. Secretaria de Ciência, Tecnologia e Insumos Estratégicos. Departamento de Ciência e Tecnologia. Diretrizes metodológicas: elaboração de pareceres técnico-científicos / Ministério da Saúde, Secretaria de Ciência, Tecnologia e Insumos Estratégicos, Departamento de Ciência e Tecnologia. 3ª ed. Brasília: Ministério da Saúde, 2011.

4. BRASIL. Ministério da Saúde. Secretaria de Ciência, Tecnologia e Insumos Estratégicos. Departamento de Gestão e Incorporação de Tecnologias em Saúde. Entendendo a Incorporação de Tecnologias em Saúde no SUS: como se envolver [recurso eletrônico] / Ministério da Saúde, Secretaria de Ciência, Tecnologia e Insumos Estratégicos, Departamento de Gestão e Incorporação de Tecnologias em Saúde. Brasília: Ministério da Saúde, 2016; 8 p.

5. BRASIL. Ministério da Saúde. Secretaria De Ciência, Tecnologia, Inovação e Insumos Estratégicos Em Saúde Departamento de Gestão e Incorporação de Tecnologias e Inovação em Saúde Coordenação-geral de Gestão de Tecnologias em Saúde Coordenação de Gestão de Protocolos Clínicos e Diretrizes Terapêuticas. Protocolo De Uso Do Distrator Osteogênico Mandibular. Brasília, 2020.

6. BRASIL. Comissão Nacional de Incorporação de Tecnologias no Sistema Único de Saúde. Protocolos e Diretrizes. Brasília: CONITEC, 2021. Disponível em http://conitec.gov.br/index.php/protocolos-e-diretrizes. Acesso em: 21 jun 2021.

7. CARDOSO DFB, et al. Traducción, adaptación cultural y validación preliminar de instrumentos para educadores de enfermería portugueses sobre práctica basada en la evidencia. Revista Enfermería Actual en Costa Rica, 2020; 38(1).

8. CARVALHO LR, ZEM-MASCARENHAS SH. Construção e validação de um cenário de simulação sobre sepse: estudo metodológico. Revista da Escola de Enfermagem da USP, 2020; 54 e03638.

9. CATUNDA HLO, et al. Methodological Approach in Nursing Research For Constructing And Validating Protocols. Texto \& Contexto - Enfermagem, 2017;26(2).

10. CONSELHO FEDERAL DE ENFERMAGEM (COFEN). Diretrizes Para Elaboração De Protocolos de Enfermagem na Atenção Primária à Saúde pelos Conselhos Regionais. Brasília, 2018.

11. CRUZ DRS, et al. Cateter de gastrostomias em crianças: elaboração de protocolos operacionais padrão. Revista Enfermagem Atual In Derme, 2019; 89(27).

12. DOMINGUES CSB, et al. Protocolo Brasileiro para Infecções Sexualmente Transmissíveis 2020: sífilis congênita e criança exposta à sífilis. Epidemiologia e Serviços de Saúde, 2021; 30(spe1) e2020597.

13. FERNANDES MA, et al. Acidentes laborais e a construção coletiva de um protocolo assistencial. Rev. enferm. UFPE online, 2019; 511-517.

14. FERRAZ L, et al. Ensino e aprendizagem da prática baseada em evidências nos cursos de Enfermagem e Medicina. Revista Brasileira de Estudos Pedagógicos, 2020; 101(257).

15. GOMES PPS, et al. Water balance in pediatric nephrology: construction of a Standard Operating Procedure. Revista Brasileira de Enfermagem, 2018; 71(suppl 3): 1404-1411.

16. GONÇALVES GAA, et al. Percepções de facilitadores sobre as tecnologias em saúde utilizadas em oficinas educativas com adolescentes. REME rev. min. enferm, 2020; e-1273.

17. JÚNIOR CL. Políticas, epidemiologia e práxis no Sistema Único de Saúde. Cadernos de Saúde Pública, 2020; 36(11): e00295120.

18. LANDEIRO MJL, et al. Evaluation of the educational technology "Caring for dependent people" by family caregivers in changes and transfers of patients and tube feeding. Revista Latino-Americana de Enfermagem, 2016; 24(0).

19. LEMOS CS, et al. Construction and validation of a nursing care protocol in anesthesia. Revista Latino-Americana de Enfermagem, 2017; 25(0).

20. MACHADO LDS, et al. Construção de Tecnologia Educativa Para Padronização De Procedimentos Operacionais da Consulta de Enfermagem. Anais do CIET:EnPED:2020 - (Congresso Internacional de Educação e Tecnologias | Encontro de Pesquisadores em Educação a Distância). 2020.

21. MERHY EE. A cartografia do trabalho vivo. Hucitec [internet], 2017; 24(8):1953-7. 
22. MORAES LMS, et al. Uso de tecnologia leve-dura nas práticas de enfermagem: análise de conceito. Aquichan, 2016; 16(2): 230-239.

23. PAIXÃO DPSS, et al. Adhesion to patient safety protocols in emergency care units. Revista Brasileira de Enfermagem, 2018; 71(suppl 1): 577-584.

24. PEREIRA LR, et al. Avaliação De Procedimentos Operacionais Padrão Implantados em um Serviço de Saúde. Arquivos de Ciências da Saúde, 2017; 24(4): 47-51.

25. PIMENTA C, et al. Guia para construção de protocolos assistenciais de enfermagem [Internet]. São Paulo: CORENSP, 2015.

26. RIBEIRO LL. A importância da identificação precoce da sepse pela equipe de enfermagem no serviço de emergência. Pub saúde, 2020; 3: a024.

27. SALES CB, et al. Standard Operational Protocols in professional nursing practice: use, weaknesses and potentialities. Revista Brasileira de Enfermagem, 2018; 71(1): 126-134.

28. SANTOS ZMSA, et al. Tecnologias em saúde: da abordagem teórica a construção e aplicação no cenário do cuidado. Fortaleza: Ed UECE, 2016

29. SANTOS NO, et al. Development and validation a nursing care protocol with educational interventions for family caregivers of elderly people after stroke. Revista Brasileira de Enfermagem, 2020; 73(suppl 3): e20180894.

30. SANTOS VT, MINAYO MCS. Mães que cuidam de crianças dependentes de tecnologia em atendimento domiciliar. Physis: Revista de Saúde Coletiva, 2020; 30(4): e300406.

31. SAUNDERS H, VEHVILÄINEN-JULKUNEN K. Nurses' Evidence-Based Practice Beliefs and the Role of EvidenceBased Practice Mentors at University Hospitals in Finland: Nurses' EBP Beliefs and Role of EBP Mentors. Worldviews on Evidence-Based Nursing, 2017; 14(1): 35-45.

32. SCHWEITZER G, et al. Implementation of the protocol of nursing care in trauma in aeromedical service. Revista Brasileira de Enfermagem, 2020; 73(3): e20180516.

33. SILVA MCDA, et al. A ciência e a tecnologia pelo olhar de gestores municipais de saúde e articuladores de saúde do idoso de uma região no interior do estado de São Paulo. Revista Tecnologia e Sociedade, 2017; 13(28).

34. TEIXEIRA SIN, et al. Procedimento Operacional Padrão na Assistência Nutricional ao Paciente com Covid 19: Relato De Experiência. Revista Ciência Plural, 2020; 6(2): 156-169.

35. VIEIRA TW, et al. Validation methods of nursing protocols: an integrative review. Revista Brasileira de Enfermagem, 2020; 73(suppl 5): e20200050. 